\title{
GPR78 promotes lung cancer cell migration and metastasis by activation of Gaq-Rho GTPase pathway
}

\author{
Dan-Dan Dong ${ }^{1}$, Hui $\mathrm{Zhou}^{2}$ \& Gao $\mathrm{Li}^{3, *}$ \\ ${ }^{1}$ Department of Pathology, Sichuan Academy of Medical Sciences, Sichuan Provincial People's Hospital, Chengdu 610072, Sichuan, \\ ${ }^{2}$ Department of Thoracic Medicine, The Affiliated Cancer Hospital of Xiangya School of Medicine, Central South University, Changsha \\ 410013, Hunan, ${ }^{3}$ Thoracic Surgery, Hainan General Hospital, Haikou 570311, Hainan, PR China
}

\begin{abstract}
GPR78 is an orphan G-protein coupled receptor (GPCR) that is predominantly expressed in human brain tissues. Currently, the function of GPR78 is unknown. This study revealed that GPR78 was expressed in lung cancer cells and functioned as a novel regulator of lung cancer cell migration and metastasis. We found that knockdown of GPR78 in lung cancer cells suppressed cell migration. Moreover, GPR78 modulated the formation of actin stress fibers in A549 cells, in a RhoA- and Rac1-dependent manner. At the molecular level, GPR78 regulated cell motility through the activation of Goq-RhoA/ Rac1 pathway. We further demonstrated that in vivo, the knockdown of GPR78 inhibited lung cancer cell metastasis. These findings suggest that GPR78 is a novel regulator for lung cancer metastasis and may serve as a potential drug target against metastatic human lung cancer. [BMB Reports 2016; 49(11): 623-628]
\end{abstract}

\section{INTRODUCTION}

G-protein-coupled receptors (GPCRs or GPRs) are among the largest and most diverse membrane protein families in mammalian genomes (1). These receptors contain seven transmembrane helices with an extracellular $\mathrm{N}$ terminus and an intracellular $\mathrm{C}$ terminus. Activated GPCRs transduce extracellular stimuli to give intracellular signals, through interaction of their intracellular domains with heterotrimeric $G$ proteins. Till date, 18 different human $\mathrm{G}$ protein $\alpha$ subunits (G $\alpha$ proteins) were identified to be coupled with GPCRs $(2,3)$. Depending on the $\alpha$ subunit type when GPCRs are activated, these $\mathrm{G} \alpha$ proteins can dissociate from the $\beta$ and $\gamma$ subunits to affect intracellular signaling proteins or target functional

*Corresponding author. Tel: +86-0898-68642221; Fax: +86-89868642222; E-mail: drligao117@163.com

https://doi.org/10.5483/BMBRep.2016.49.11.133

Received 4 August 2016, Revised 25 August 2016, Accepted 23 September 2016

Keywords: GPR78, Lung cancer, Metastasis, Rho GTPase proteins (1). For most GPCRs, the physiologic function is currently unknown, and these receptors are referred to as orphan GPCRs (4). GPR78 is an orphan GPCR first identified by sequence comparison with GPR26 (5). Expression analysis revealed it was highly expressed in human brain tissue. Later studies demonstrated that GPR78 overexpression in HEK293 cells was constitutively active, and induced the cAMP production (6). However, the cellular function of GPR78 remains unknown.

The Rho family GTPases, including RhoA, Rac1, and Cdc42, are a family of small $(\sim 21 \mathrm{kDa})$ signaling $\mathrm{G}$ proteins $(7)$. They are regulators of actin cytoskeleton rearrangements, and thus modulate cell migration (8). For example, RhoA regulates stress fiber formation, and Rac1 modulates a meshwork of actin filaments at the cell periphery to produce lamellipodia and membrane ruffles (9). Rho proteins act as switches by alternating between inactive guanosine diphosphate (GDP)bound and active guanosine triphosphate (GTP)-bound states. This process can be accelerated by a large family of Rho guanine nucleotide exchange factors (10). GPCR can activate Rho GTPases by coupling Rho-GEFs through their heterotrimeric guanine nucleotide-binding protein. This singling transduction pathway is involved in multiple physiologic functions, including cell migration and invasion $(10,11)$.

In this study, we found that GPR78 was expressed in lung cancer cells, and promoted cell migration and metastasis in a Gaq-Rho GTPase-dependent manner. Our results suggest that GPR78 is a potential regulator for lung cancer metastasis that may serve as a potential drug target for lung cancer metastasis.

\section{RESULTS}

GPR78 is expressed in lung cancer cells and promotes cell migration

GPR78 is an orphan G-protein coupled receptor (GPCR) that is highly expressed in human placenta and brain tissues (6). However, the expression pattern and function of GPR78 in human cancer cells is still unknown. To explore the role of GPR78 in cancer cells, we first examined the expression of GPR78 in several lung cancer cells by western blotting. Interestingly, we found that GPR78 expressed in several lung

ISSN: 1976-670X (electronic edition)

Copyright (C) 2016 by the The Korean Society for Biochemistry and Molecular Biology

(c) This is an open-access article distributed under the terms of the Creative Commons Attribution Non-Commercial License (http://creativecommons.org/licenses/by-nc/4.0) which permits unrestricted non-commercial use, distribution, and reproduction in any medium, provided the original work is properly cited. 
cancer cell lines including H1975, H1299, SPC-A1 and A549 cells, but not in normal human bronchial epithelial cell line such as HBEC3 and HBEC4 (Fig. 1A). Furthermore, the mRNA
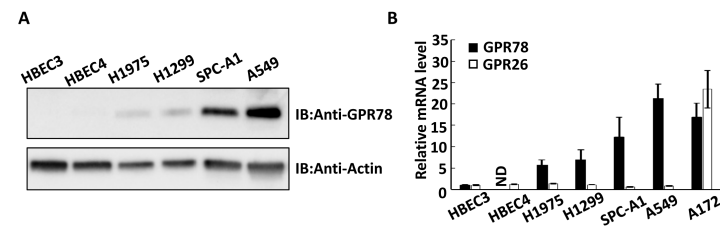

D
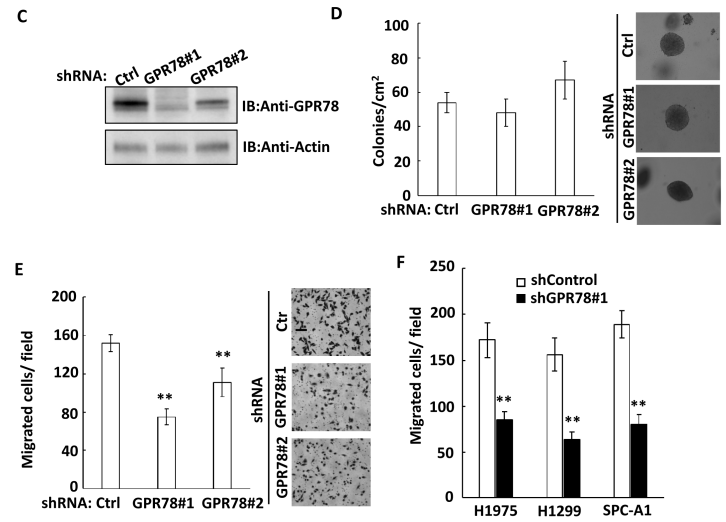

G
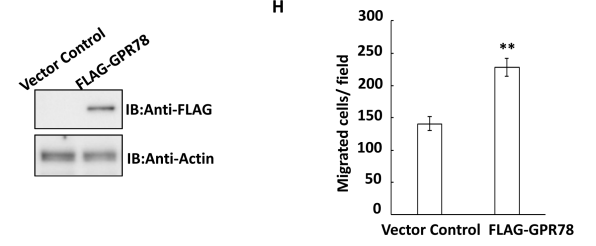

Fig. 1. GPR78 promotes lung cancer cell migration. (A) The expression of GPR78 and actin were analyzed by immunoblotting in normal human bronchial epithelial cell lines (HBEC3 and HBEC4) or lung cancer cell lines (H1975, H1299, SPC-A1 and A549). (B) The mRNA expression of GPR78 and GPR26 were analyzed by real-time PCR in normal and cancer cell lines, as indicated. ND, Not-detectable. (C) A549 cells were stably transfected with shRNA control or two shRNAs targeting GPR78 (GPR78\#1 and GPR78\#2). The expression of GPR78 and actin were analyzed by immunoblotting. (D) shRNA control, GPR78\#1 and GPR78\#2 A549 cells were seeded in soft agar and cultured for 14 days to evaluate the colony formation capability. Left, quantitative data of colony formation assays: colonies $>1 \mathrm{~mm}$ in diameter were counted. Right, representative colony images are shown. (E) shRNA control, GPR78\#1 and GPR78\#2 A549 cells were analyzed by Transwell assay for cell migration. Left, quantitative data of migrated cells. Results shown are averages \pm S.E.M. Right, representative images of Transwell assay. (F) H1975, H1299 or SPC-A1 lung cancer cell lines were stably transfected with shRNA control or shRNA GPR78\#1. Cell migration was analyzed by Transwell assay. Quantitative data of migrated cells are shown. Results shown are averages \pm S.E.M. (G) A549 cells were transfected with vector control or FLAG-GPR78, and the expression of FLAG-GPR78 and actin were analyzed by immunoblotting. (H) Vector control or FLAG-GPR78 A549 cell migration was analyzed by Transwell assay. Quantitative data of migrated cells are shown. Results shown are averages \pm S.E.M. ${ }^{* * P}<0.01$, compared with control. level of GPR78 was also dramatically increased in lung cancer cells but not in normal cells (Fig. 1B). Since GPR26 shares more than $50 \%$ sequence similarity with GPR78 (6), we then examined the mRNA expression of GPR26 in these cells by real-time PCR. As shown in Fig. 1B, the mRNA level of GPR26 was extremely low in both normal and lung cancer cells, when compared with the human brain glioma cell line A172 cells, which was used as a positive control for detecting the expression of GPR78 and GPR26. To investigate the cellular function of GPR78 in lung cancer cells, we then established two stable shRNA-mediated knockdown cell lines in A549 cells (Fig. 1C), in which GPR78 was highly expressed (Fig. 1A and B). We observed that knockdown of GPR78 did not influence the number and size of the colonies in the colony formation assay (Fig. 1D). However, the knockdown of GPR78 significantly inhibited cell migration, as measured by the Transwell migration assay (Fig. $1 \mathrm{E}$ ). In addition, this inhibitory effect on cell migration was also confirmed in other metastatic lung cancer cell lines, including H1975, H1299 and SPC-A1 cells (Fig. 1F). To further confirm the role of GPR78 in promoting cell migration, we ectopically overexpressed GPR78 in A549 cells (Fig. 1G), and found that overexpression of GPR78 promoted cell migration (Fig. 1H). Collectively, our data suggests that GPR78 expresses in lung cancer cells and promotes the cell migration.

\section{GPR78 regulates cell migration by targeting RhoA- and Rac1-dependent pathways}

We observed that GPR78 knockdown A549 cells exhibited an elongated morphology compared to shRNA control cells (Fig. $2 A$, Left). Quantification of these cells indicated that the percentage of elongated cells significantly increased by GPR78 knockdown in A549 cells (Fig. 2A, right). Since elongation of cell morphology is related to the reduction of stress fibers (F-actin) (12), we stained the stress fibers in both control and GPR78 shRNA cells. As shown in Fig. 2B, knockdown of GRP78 decreased stress fibers in A549 cells. It has been reported that small GTPase RhoA and Rac1 play an important role in regulating cellular morphology and stress fiber formation (13); therefore, our next query was whether GPR78 regulates the activity of RhoA and Rac1. Interestingly, evaluating by GTPase assay, we found that knockdown of GPR78 significantly suppressed the RhoA and Rac1 activity in A549 cells (Fig. 2C). Importantly, overexpression of constitutive active RhoA or Rac1 in GPR78-knockdown A549 cells partially restored cell migration (Fig. 2D and E). Collectively, our data suggests that GPR78 regulates cell morphology and migration by modulating the activity of RhoA and Rac1 in lung cancer cells.

\section{Gaq/11 mediates GPR78-induced activation of RhoA and Rac1}

Previous reports have shown that $\mathrm{G} \alpha 12 / 13$ and $\mathrm{G} \alpha \mathrm{q} / 11$ are the major G $\alpha$ proteins involved in activation of the Rho family 

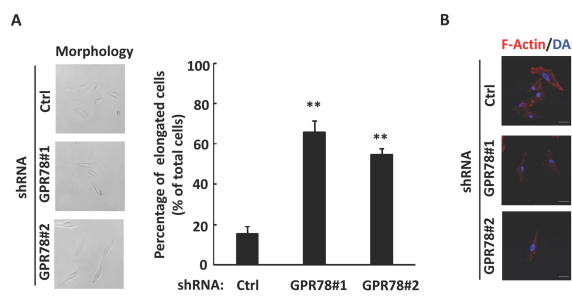
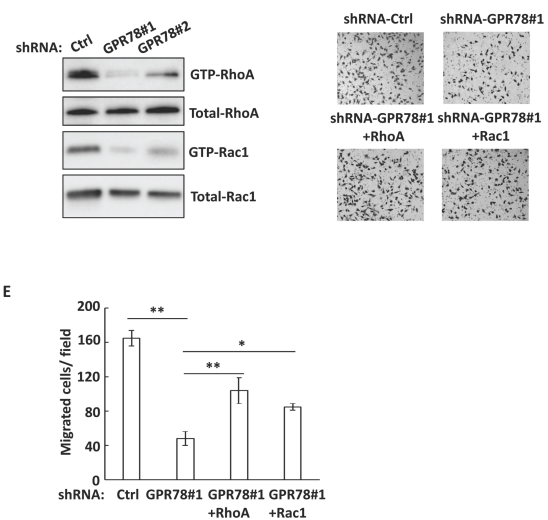

Fig. 2. GPR78 regulates cell morphology by activating small GTPases. (A) Left, shRNA control, GPR78\#1 and GPR78\#2 A549 cells were photographed by phase-contrast microscopy and representative images are shown. Magnification, $\times 200$. Right, number of elongated cells was counted. (B) The cells were stained with Alexa Fluor 594 phalloidin (red) for F-actin and DAPI (blue) for nuclear stain. Representative images are shown. Magnification, $\times 600$. (C) Knockdown of GPR78 inhibits the activation of RhoA and Rac1 in A549 cells by GTPase assay. (D) shRNA-GPR\#1 A549 cells were transfected with constitutively active mutant RhoA Q63L or Rac1 Q61L, and the ability for cell migration was examined by Transwell migration assay. Representative images of Transwell migration assay are shown. Magnification, $\times 100$. (E) Quantitative data of migrated cells in (D). Results shown are averages \pm S.E.M. ${ }^{* * P}<0.01$

GTPases (14-16). Similar to our previous conclusion (Fig. 2C), we found that overexpression of GPR78 in A549 cells induced the activation of RhoA and Rac1 (Fig. 3A). To investigate whether G $\alpha 12 / 13$ or Gaq/11 mediates GPR78-induced activation of RhoA and Rac1, we co-expressed GPR78 with RGS domains of $p 115$ Rho-GEF regulator ( $p 115-R G S)$ or GRK2 (GRK2-RGS), which selectively block the activity of G $\alpha 12 / 13$ or Gaq/11 (17, 18), respectively. We found that GPR78induced activation of RhoA and Rac1 was inhibited by co-expression of GRK2-RGS, but not p115-RGS (Fig. 3A). Moreover, overexpression of a constitutively active mutant of Gaq/11 (CA Gq) in GPR78 knockdown A549 cells partially restores the activity of RhoA and Rac1 disrupted by GPR78 knocking down (Fig. 3B). Thus, these data suggest that Gaq/11 mediates the GPR78-induced activation of RhoA and Rac1. To further investigate whether GPR78-regulated cell migration was dependent on Gaq/11, we expressed the active mutant of
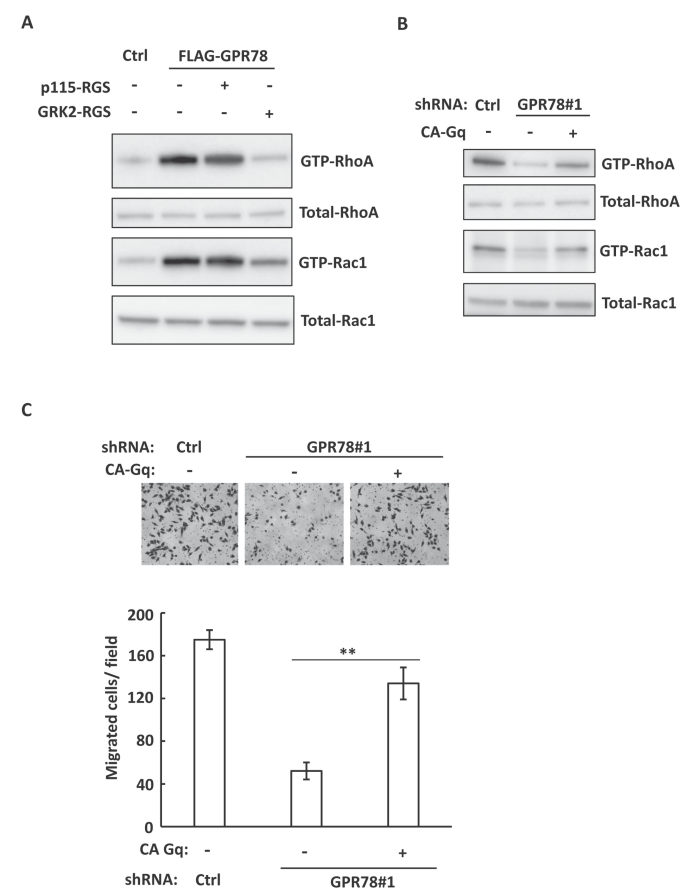

Fig. 3. G $\alpha q / 11$ signaling pathway mediates GPR78-regulated cell invasion. (A) Control or FLAG-GPR78 overexpressed A549 cells were transfected with G $\alpha 12 / 13$ inhibitor p115-RGS, or G $\alpha q / 11$ inhibitor GRK2-RGS, as indicated. The activation of RhoA and Rac1 was analyzed by GTPase assay. (B) shRNA-control or shRNA-GPR78\#1 A549 cells were transfected with constitutive Gaq/11 protein CA-Gq, as indicated. The activation of RhoA and Rac1 was analyzed by GTPase assay. (C) The cells used in (B) were analyzed for invasion ability by Transwell migration assay. Upper panel, Representative images of Transwell assay. Lower panel, Quantitative data of migrated cells are shown. Results shown are averages \pm S.E.M.

Gaq/11 in GPR78 knockdown A549 cells, and analyzed the cell migration. As shown in Fig. 3C, expression of CA Gq partially rescued cell migration in GPR78 knocking down cells. Thus, these data indicate that G $\alpha \mathrm{q} / 11$ mediates GPR78regulated cell migration by activation of RhoA and Rac1.

\section{Knockdown of GPR78 in lung cancer cells suppresses metastasis in vivo}

To examine whether GPR78 regulates lung cancer cell growth and metastasis in vivo, we investigated lung metastasis of A549 cells using xenograft mouse model. We discovered that GPR78 knockdown in A549 cell did not influence the tumor growth in vivo (Fig. 4A and B). However, knockdown of GPR78 significantly suppressed lung metastasis in vivo (Fig. 4C and D). Taken together, these data suggest that GPR78 regulates breast tumor cell metastases in vivo. 
A

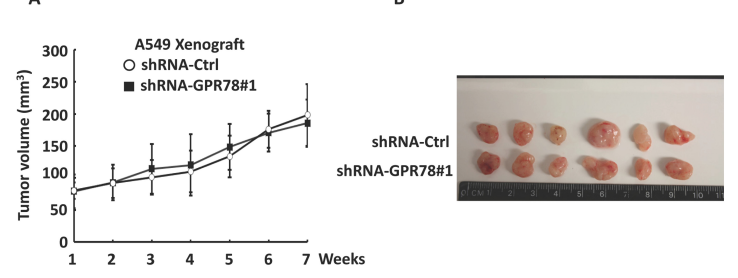

c

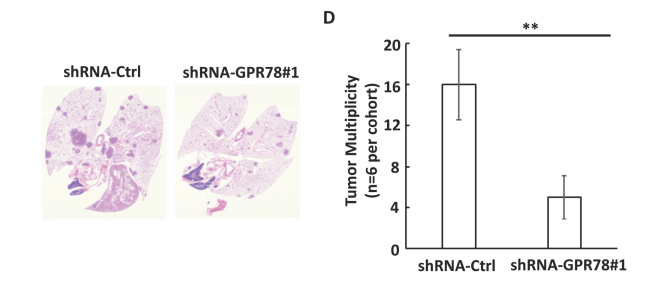

Fig. 4. Knockdown of GPR78 inhibits lung tumor metastasis in vivo. (A) Control or GPR78\#1 shRNA A549 cells were injected s.c. into 6-week-old female nude mice. Tumor growth curve or (B) final images for A549 tumor xenografts are shown. (C) Representative $\mathrm{H} \& \mathrm{E}$ images of lungs of mice from (A). (D) Lung tumor multiplicity of mice from (A) was determined by counting total tumor foci in 5 serial sections at $400 \mathrm{~mm}$ intervals. ${ }^{* *} \mathrm{P}<0.01$.

\section{DISCUSSION}

GPR78 is an orphan G-protein coupled receptor (GPCR), highly expressed in human placenta and brain tissues (6). However, the expression pattern of GPR78 in human cancer cells is currently unknown. In this study, we found that GPR78 was expressed in multiple human lung cancer cell lines but not in normal bronchial epithelial cells. GPR26, a paralog gene of GPR78 (6), expressed very low levels in lung cancer cells, it probably has a redundant function, since GPR78 is present in these cells. We investigated the cellular function of GPR78 by GPR78 knockdown in lung cancer cells. Interestingly, knockdown of GPR78 significantly inhibited cell migration but not cell proliferation. Although identification of endogenous ligand(s) for GPR78 will facilitate in examining its role in the cells, a previous study suggested that constitutive activation of GPR78 can be induced by overexpression of itself (6). We thus overexpressed GPR78 in A549 cells, and found that overexpression of GPR48 promoted cell migration, further confirming that GPR78 functions as a regulator for cell migration in lung cancer cells. It has been reported that certain haplotypes of GPR78 were linked to increased susceptibility to biopolar disorders (BPD) and schizophrenia (SZ) in a Scottish family (19). However, the function of GPR78 in modulating cell migration was not reported. Thus, for the first time, our results demonstrate that GPR78 expresses in lung cancer cells and functions as a regulator for cell migration.

Subsequently, we investigated the molecular mechanism of GPR78 in regulating cell migration. We found that knockdown of GPR78 significantly suppressed activity of RhoA and Rac1, while overexpression of GPR78 induced RhoA and Rac1 activity. Previous studies demonstrated that RhoA and Rac1 modulate elongation of cell morphology, and regulate cancer cell migration $(20,21)$. Our study further presented that RhoA and Rac1 are responsible for GPR78-regulated cell migration. Moreover, GPR78-induced activation of RhoA and Rac1 is mediated by Gaq-signaling pathway, since blocking of Gaq-signaling inhibited the activation of RhoA and Rac1. It has been reported that GPR78 is a Gs-coupled receptor in regulating cellular level of cAMP. Our study indicates that GPR78 is involved in cell migration through the classical G $\alpha$ protein-mediated signaling pathway. We observed that the ectopic expression of active mutant CA Gq partially rescued cell migration in GPR78 knockdown cells. These results suggested that other downstream targets of GPR78 may also contribute to GPR78-regulated cell migration. Several GPCRs are known to regulate tumor progression by coupling to the $\mathrm{G} \alpha$ protein signaling pathway. For example, GPR56 is involved in the progression of pancreatic cancer and glioblastoma by coupling to $\mathrm{G} \alpha 12 / 13$ and $\operatorname{G\alpha q} / 11(22,23)$. CD97 promoted thyroid cancer progression by coupling to Ga12/13 (16). GPR116 promoted breast cancer metastasis by activating Gaq-Rho GTPase pathway (24). Thus, our study indicates that GPR78 can couple with the classical Gaq protein to mediate cancer cell migration and metastasis in lung cancer.

Collectively, this study is the first to demonstrate that GPR78 expresses in lung cancer cells, and plays an important role in promoting tumor cell migration and metastasis. Mechanistic studies identified that GPR78 activates RhoA and Rac1 by coupling to the Gaq-mediated signaling pathway. Importantly, a mouse xenograft model further demonstrated that knockdown of GPR78 inhibited lung cancer cell metastasis in vivo, confirming that GPR78 is a novel regulator for lung cancer metastasis, and may serve as a potential drug target against metastatic human lung cancer.

\section{MATERIALS AND METHODS}

\section{Reagents}

Antibodies to GPR78 (Cat. No. ab121390), RhoA (Cat. No. ab187027), Rac1 (Cat. No. ab33186) and $\beta$-actin (Cat. No. ab8226) were purchased from Abcam (Cambridge, MA, USA). Antibodies to FLAG (Cat. No. F7425) were purchased from Sigma-Aldrich (St. Louis, MO, USA). Alexa Fluor ${ }^{R} 568$ Phalloidin was purchased from ThermoFisher Scientific (Grand Island, NY, USA).

\section{Cell culture}

HBEC3, HBEC4, A549, H1975, H1299, SPC-A1 and A172 cell lines were purchased from ATCC. Cells were maintained in DMEM medium (Invitrogen, USA) supplemented with 10\% fetal bovine serum, 100 units $/ \mathrm{ml}$ penicillin $\mathrm{G}, 100 \mu \mathrm{g} / \mathrm{ml}$ streptomycin, and $2 \mathrm{mM} \mathrm{L-glutamine,} \mathrm{at} 37^{\circ} \mathrm{C}$ with $5 \% \mathrm{CO}_{2}$. 
Immortalized HBEC3 and HBEC4 cells were cultured in Keratinocyte Serum Free media (KSFM) (Gibco) at $37^{\circ} \mathrm{C}$ and $5 \% \mathrm{CO}_{2}$. The KSFM media contained $50 \mu \mathrm{g} / \mathrm{ml}$ of bovine pituitary extract and $5 \mu \mathrm{g} / \mathrm{ml}$ of epidermal growth factor.

\section{Western blotting}

Cells were lysed in $\mathrm{M} 2$ lysis buffer $(150 \mathrm{mM} \mathrm{NaCl}, 50 \mathrm{mM}$ Tris-Cl (pH 8.0), 5 mM EDTA, $1 \%$ Nonidet P-40) containing a protease inhibitor mixture (Roche Applied Science) and a phosphatase inhibitor mixture (Sigma, MO, USA). Equal amounts of total protein were subjected to SDS-PAGE analysis and immunoblotting with the appropriate antibodies.

\section{Real-time PCR assay}

RNA was extracted using TRIzol (ThermoFisher, Grand Island, USA) and quantified by spectrophotometry (NanoDrop, Peqlab, Germany). RNA was reverse transcribed using Superscript III reverse transcriptase (ThermoFisher, Grand Island, USA), according to the manufacturer's protocol. PCR was conducted using the MyiQ Real-Time PCR Detection System (Bio-Rad, USA). The Taqman probe for gpr78 (Assay ID: Hs01574416 m1), gpr26 (Hs00538034_m1), and actin (Assay ID: Hs.PT.56a.21538384) were purchased from Thermo Scientific (Waltham, MA, USA). The threshold cycle number for each gene was normalized to that of actin, and the resulting value was converted to a linear scale.

\section{Plasmid construction and transfection}

Full-length DNA coding sequence of human GPR78 (NM 080819) was amplified using the forward primer 5'GGATCCCATGGGCCCCGGCGAG-3' and the reverse primer 5'-GTCGACGCCAGGCCCTCAGTGTG-3. The amplified PCR product was then cloned into the $\mathrm{BamH} 1 /$ Sal1 site of pCMV-Tag2A construct and verified by DNA sequencing. Both p115-RGS (amino acid residues 1-252) and GRK2-RGS (amino acid residues 54-175) were amplified by PCR and sub-cloned into the BamH1/ EcroR1 site of pCMV-Tag2A construct. The constitutively active mutants of RhoA, Rac1, and Gq were cloned as previously described (25).

The lentivirus silencing vector expressing shRNA targeting human GPR78\#1 (TRC number: TRCN0000357332) and GPR78\#2 ADAM10 (TRC number: TRCN0000357333) were obtained from Sigma (St. Louis, MO, USA). Following the manufacturer's instructions, plasmids were transfected into cells using lipofectamine 2000 (ThermoFisher Scientific, Grand Island, USA). For lentivirus production, HEK293T cells were co-transfected with pCMV-VSV-G, pCMV-dr8.2-dvpr and shRNA plasmids. After $24 \mathrm{~h}$, the supernatant was collected and this lentiviral preparation was used to infect cells. After 24 $\mathrm{h}$ of infection, cell selection was accomplished by incubating with puromycin for an additional $48 \mathrm{~h}$.

\section{GTPase activity assay}

For RhoA activity, a fusion protein of glutathione S-transferase
(GST) with the RhoA binding domain of Rhotekin was used (GST-RBD). For Rac1 activity, a GST fusion protein with the binding domain of PAK was used (GST-PBD). Briefly, both fusion proteins expressed in BL21 were purified with glutathione Sepharose beads (GE Healthcare). Cell lysates were prepared with RhoA lysis buffer $[50 \mathrm{mmol} / \mathrm{L}$ Tris- $\mathrm{HCl}$, $\mathrm{pH} 7.4,150 \mathrm{mmol} / \mathrm{L} \mathrm{NaCl}, 5 \mathrm{mmol} / \mathrm{L} \mathrm{MgCl} 2,1 \mathrm{mmol} / \mathrm{L}$ dithiothreitol, $1 \mathrm{mmol} / \mathrm{L}$ phenylmethylsulfonyl fluoride (PMSF), $1 \%$ Triton X-100, proteinase inhibitor cocktail] or Rac1 lysis buffer $(20 \mathrm{mmol} / \mathrm{L} \mathrm{HEPESNaOH}, \mathrm{pH} 7.5,120 \mathrm{mmol} / \mathrm{L} \mathrm{NaCl}$, $10 \%$ glycerol, $2 \mathrm{mmol} / \mathrm{L}$ EDTA, $0.5 \%$ Nonidet P-40, $1 \mathrm{mmol} / \mathrm{L}$ PMSF, proteinase inhibitor cocktail], and incubated with GST-RBD or GST-PBD precoupled to glutathione Sepharose beads, at $4^{\circ} \mathrm{C}$ for 45 minutes; this precipitates the GTP-bound RhoA or Rac1. The precipitant was then boiled in Laemmli sample buffer, and the eluted product was separated on a $12 \%$ SDS-PAGE gel. Immunoblotting was used to detect the protein level of total and GTP-bound RhoA or Rac1.

\section{Transwell migration assay}

The transwell migration assay was performed using 6.5-mmdiameter polycarbonate filters $\left(8-\mu \mathrm{m}\right.$ pore size). Cells $\left(4 \times 10^{4}\right)$ suspended in $100 \mu \mathrm{l}$ of DMEM containing $0.5 \%$ FBS, were seeded in the top chambers. The bottom chambers were filled with $500 \mu \mathrm{l}$ of DMEM containing $10 \%$ FBS. Cells were allowed to migrate for 12 hours. The unmigrated cells were removed with cotton swabs. Migrated cells were fixed with cold $4 \%$ paraformaldehyde and stained with $1 \%$ crystal violet. Images were taken using an inverted microscope (10X magnification; Olympus, Tokyo, Japan), and migrated cells were quantified by manual counting.

\section{in vivo A549 xenograft model}

A549 cells $\left(2 \times 10^{6}\right)$ were mixed with matrigel and subcutaneously (s.c.) injected into nude mice ( $n=6$ for each group) under aseptic conditions. Tumors were measured in 2 dimensions, and volume was calculated according to the formula: $V=0.5$ (length $\times$ width $^{2}$ ). The tumor was monitored and evaluated every week. After 7 weeks, the tumor and lung were excised and evaluated. The total number of tumor foci was determined by five serial sections at $400 \mathrm{~mm}$ intervals.

\section{Statistical analysis}

Data were analyzed using the SPSS (version 20.0; IBM Corporation, Armonk, NY, USA) software program. Results are expressed as means \pm S.D. and are representative of at least three separate experiments. The two-sample t-test was used to determine statistical differences in the means of two columns. $P$ value less than $0.05(P<0.05)$ was regarded as statistically significant.

\section{REFERENCES}

1. Kroeze WK, Sheffler DJ and Roth BL (2003) G-protein- 
coupled receptors at a glance. J Cell Sci 116, 4867-4869

2. Hermans E (2003) Biochemical and pharmacological control of the multiplicity of coupling at G-proteincoupled receptors. Pharmacol Ther 99, 25-44

3. Wong SK (2003) G protein selectivity is regulated by multiple intracellular regions of GPCRs. Neurosignals 12, $1-12$

4. Tang XL, Wang Y, Li DL, Luo J and Liu MY (2012) Orphan G protein-coupled receptors (GPCRs): biological functions and potential drug targets. Acta Pharmacol Sin 33, 363-371

5. Lee DK, Nguyen T, Lynch KR et al (2001) Discovery and mapping of ten novel $\mathrm{G}$ protein-coupled receptor genes. Gene 275, 83-91

6. Jones PG, Nawoschik SP, Sreekumar K et al (2007) Tissue distribution and functional analyses of the constitutively active orphan G protein coupled receptors, GPR26 and GPR78. Biochim Biophys Acta 1770, 890-901

7. Hall A (2012) Rho family GTPases. Biochem Soc Trans 40, 1378-1382

8. Fukata M, Nakagawa M and Kaibuchi K (2003) Roles of Rho-family GTPases in cell polarisation and directional migration. Curr Opin Cell Biol 15, 590-597

9. Hall A (1998) Rho GTPases and the actin cytoskeleton. Science 279, 509-514

10. Lutz S, Shankaranarayanan A, Coco C et al (2007) Structure of Galphaq-p63RhoGEF-RhoA complex reveals a pathway for the activation of RhoA by GPCRs. Science 318, 1923-1927

11. Momotani K, Artamonov MV, Utepbergenov D, Derewenda U, Derewenda ZS and Somlyo AV (2011) p63RhoGEF couples Galpha(q/11)-mediated signaling to Ca2 + sensitization of vascular smooth muscle contractility. Circ Res 109, 993-1002

12. Yamaguchi $\mathrm{H}$ and Condeelis J (2007) Regulation of the actin cytoskeleton in cancer cell migration and invasion. Biochim Biophys Acta 1773, 642-652

13. Tojkander S, Gateva G and Lappalainen P (2012) Actin stress fibers-assembly, dynamics and biological roles. J Cell Sci 125, 1855-1864

14. Iguchi T, Sakata K, Yoshizaki K, Tago K, Mizuno N and Itoh $\mathrm{H}$ (2008) Orphan $\mathrm{G}$ protein-coupled receptor GPR56 regulates neural progenitor cell migration via a $G$ alpha
12/13 and Rho pathway. J Biol Chem 283, 14469-14478

15. Cotton M and Claing A (2009) G protein-coupled receptors stimulation and the control of cell migration. Cell Signal 21, 1045-1053

16. Ward Y, Lake R, Martin PL et al (2013) CD97 amplifies LPA receptor signaling and promotes thyroid cancer progression in a mouse model. Oncogene 32, 2726-2738

17. Yuan B, Cui J, Wang W and Deng K (2016) Galpha12/13 signaling promotes cervical cancer invasion through the RhoA/ROCK-JNK signaling axis. Biochem Biophys Res Commun 473, 1240-1246

18. Schumacher SM, Gao E, Cohen M, Lieu M, Kurt Chuprun J and Koch WJ (2016) A peptide of the RGS domain of GRK2 binds and inhibits Galphaq to suppress pathological cardiac hypertrophy and dysfunction. Sci Signal 9, ra30

19. Underwood SL, Christoforou A, Thomson PA et al (2006) Association analysis of the chromosome 4p-located G protein-coupled receptor 78 (GPR78) gene in bipolar affective disorder and schizophrenia. Mol Psychiatry 11, 384-394

20. Gulhati P, Bowen KA, Liu J et al (2011) mTORC1 and mTORC2 regulate EMT, motility, and metastasis of colorectal cancer via RhoA and Rac1 signaling pathways. Cancer Res 71, 3246-3256

21. Zhao P, Zhang W, Wang SJ et al (2011) HAb18G/CD147 promotes cell motility by regulating annexin II-activated RhoA and Rac1 signaling pathways in hepatocellular carcinoma cells. Hepatology 54, 2012-2024

22. Shashidhar S, Lorente G, Nagavarapu U et al (2005) GPR56 is a GPCR that is overexpressed in gliomas and functions in tumor cell adhesion. Oncogene 24, 16731682

23. Huang Y, Fan J, Yang J and Zhu GZ (2008) Characterization of GPR56 protein and its suppressed expression in human pancreatic cancer cells. Mol Cell Biochem 308, 133-139

24. Tang X, Jin R, Qu G et al (2013) GPR116, an adhesion G-protein-coupled receptor, promotes breast cancer metastasis via the Galphaq-p63RhoGEF-Rho GTPase pathway. Cancer Res 73, 6206-6218

25. Johansson BB, Minsaas L and Aragay AM (2005) Proteasome involvement in the degradation of the $\mathrm{G}(\mathrm{q})$ family of Galpha subunits. FEBS J 272, 5365-5377 